\title{
CLINICOEPIDEMIOLOGICAL PROFILE AND POOR PROGNOSTIC FACTORS IN NEUROTUBERCULOSIS: STUDY OF INDIAN CHILDREN
}

\author{
P. Sharma, B. Verma \\ Pediatrics, Sir JJ Group of Hospitals, Mumbai, India
}

Background: Neurotuberculosis is associated with high mortality and morbidity and is difficult to diagnose , has variable presentation and difficult to treat.

Aims: To study the clinical, demographic and diagnostic parameters and independent poor prognostic factors in children with neurotuberculosis.

Methods: We prospectively reviewed all cases of neurotuberculosis diagnosed over a period of 19 months. Data were collected with regard to the clinical, laboratory and demographic characteristics of patients, as well as the results of radiological investigations and data on clinical outcome.

Results: 50 patients of neurotuberculosis were enrolled in the present study. 40 cases of tuberculous meningitis , 4 cases of tuberculous meningitis with tuberculoma and 6 cases of tuberculoma. The age of presentation varied from 1 month to 11years. Out of 44 patients of tuberculous meningitis $4(9.09 \%)$ presented in Stage 1 of TBM , $25(56.82 \%)$ in stage 2 and $15(34.09 \%)$ in stage 3. Patients with absence of BCG scar presented in higher stage of TBM $(p$ value $=0.048)$. In this study 11 patients died $(22 \%)$. Presence of focal neurological deficits ( $p$ value $=0.031)$, stage of TBM $(p$ value $=0.002)$ and HIV positive status $(p$ value $=0.031)$ were found to significantly affect mortality.

Conclusion: Neurotuberculosis continues to be a condition which carries significant morbidity and mortality. Focal neurological deficits, HIV positive status, stage of TBM and BCG unimmunised status are independent predictors of poor prognosis in patients of neurotuberculosis. 